\title{
NECROTIZING HISTIOCYTIC LYMPHADENITIS/KIKUCHI-FUJIMOTO DISEASE: A CASE REPORT AND REVIEW OF LITERATURE
}

\author{
Srinivas Gubbala ${ }^{1}$, V. Satyanarayana ${ }^{2}$
}

\section{HOW TO CITE THIS ARTICLE:}

Srinivas Gubbala, V. Satyanarayana. "Necrotizing Histiocytic Lymphadenitis/Kikuchi-Fujimoto Disease: A case report and review of Literature". Journal of Evolution of Medical and Dental Sciences 2014; Vol. 3, Issue 48, September 29; Page: 11617-11621, DOI: 10.14260/jemds/2014/3524

ABSTRACT: Kikuchi-Fujimoto disease is a rare benign, condition of necrotizing histiocytic lymphadenitis. A case of a 21 year old gentleman is described here. He presented with fever, weight loss and non-tender cervical lymph nodes. Kikuchi-Fujimoto disease was diagnosed after cervical lymph node biopsy. Symptomatic treatment was provided and an uneventful full recovery was made. BACKGROUND: The presenting complaint of neck masses in association with non-specific systemic signs and symptoms prompt investigation towards the more common diagnoses. However, rarer conditions like Kikuchi-Fujimoto disease must be included in the differential diagnosis of necrotizing lymphadenopathies such as tuberculosis, lupus erythematosus and lymphoma as its course and treatment are entirely different. (1)Here in we discuss a case of male patient who presented with a neck mass that was not attributable to the more common causes.

KEYWORDS: Cervical lymphadenopathy kikuchi-fujimoto disease.

CASE PRESENTATION: A 21 year old male patient presented with a two week history of fever, anorexia and weight loss. There were no other complaints. He had been previously fit and well and was on no medication. On examination, he was lethargic, but otherwise looked well. He was afebrile and hemodynamically stable. Significant findings were generalized lymphadenopathy, palpable in the cervical and axillary regions.

There was no evidence of hepatosplenomegaly. Blood tests revealed neutropenia, hyponatraemia, raised alkaline phosphate and C-reactive protein. Electrocardiogram and chest radiographs were normal. His initial management consisted of fluid restriction and regular paracetamol, whilst results of further tests were awaited. These included blood and sputum cultures, autoimmune and viral screens.

Subsequently there was a reduction in size and tenderness of the cervical lymph nodes. There were persistent intermittent temperature spikes and a two day episode of self-resolved diarrhea.

Blood, urine and stool cultures were negative. Sputum cultures grew respiratory tract flora and were negative for acid-fast bacilli. The autoimmune screen was negative, as was toxoplasma and cytomegalovirus screens.

Computerized tomography demonstrated cervical and axillary lymphadenopathy The abdominal viscera were normal.

Excisional biopsy of a cervical lymph node confirmed a diagnosis of Kikuchi-Fujimoto disease (KFD). Histological analysis showed histiocytic granulomatous infiltration with widespread paracortical necrosis of the lymph node, extensive karyorrhectic debris and scattered fibrin deposits and sheets of foam cells. Special stains for acid fast bacilli (Ziehl-Neelsen) and fungi (Gomori's methanamine silver and periodic acid Schiff with diastase) did not reveal any micro-organisms. 
Mycobacterial cultures and polymerase chain reaction (PCR) for Mycobacterium tuberculosis could not be performed.

No active treatment was initiated and the patient was discharged home after a one week hospital stay.

At follow -up he reported no symptoms, remained well and there were no abnormalities on clinical examination.

DISCUSSION: KFD is a benign histiocytic necrotizing lymphadentitis. KFD is rare, but common in Asia. In the early 1970 both Kikuchi and Fujimoto first described cases of KFD in Japan.(2,3) Its etiology has not yet been fully determined, however it is believed that it may be of viral origin, EBV, HHV6 and 8 have been suggested. Raw fish was postulated as a cause, but the recent literature doesn't support this. (4)

An autoimmune etiology is also likely as it has been reported in association with SLE. It tends to affect a young population under 30 years of age, including children, although the latter are less commonly affected. There are reported cases in older age group and pregnant women too (5).Early reports suggest affected female cases are more common; however more recently this view has changed to one of equal prevalence in both genders.

The most common signs and symptoms are lymphadenopathy, fever, sweats, malaise, anorexia, weight loss, hepatomegaly and leucopenia.(4) The viral etiology of KFD is supported by its non-specific self-resolving symptoms, which are of slow, insidious onset. A definite diagnosis is made by tissue biopsy, indeed whole lymph node biopsy. Histopathological assessment of affected lymph nodes reveals characteristic findings.

There are three main patterns identified, proliferative, necrotizing and xanthomatous. The proliferative picture is seen in approximately a third of cases and has a dominant inflammatory infiltrate. Half of cases show necrotizing pattern and the xanthomatous type is rare and has abundant foam cells.(6) Cellular protein structures have been noted in the cytoplasm of lymphocytes and histiocytes that have also been found in those cells of patients with SLE. This adds strength to the hypothesis that KFD is a self-limiting SLE-like disorder.

As the symptoms are non-specific and some of the histological features are similar to other diseases it is easy to misdiagnose KFD with SLE or lymphoma. This is important as the treatment of KFD is symptomatic and supportive, spontaneous recovery is usual, while the latter two conditions require prompt specific treatments.

Long term follow-up of these patients is necessary as recurrent case of KFD have been reported and there is some belief that KFD may be a precursor for SLE, as both diseases have had concurrent and co-existing disease patterns in the same patients.(7-9) In a review of KFD cases by Kucukardali et al the reported overall mortality rate associated with KFD is $2.1 \%$.(10)

CONCLUSION: KFD is uncommon, but should feature in a list of differential diagnoses of non-tender lymphadenopathy, especially affecting the cervical region. Its treatment differs significantly from other conditions such as SLE, lymphoma and TB. Lymph node biopsy will aid in accurate diagnosis, but if confusion with SLE occurs, differentiation can be made with the aid of blood tests for complement levels amongst others. Recurrence has been reported. Long term follow up is warranted. 


\section{LEARNING POINTS:}

- KFD is an important diagnosis to make treatment is conservative and this differs with the management of other conditions it may be confused with.

- KFD requires a tissue diagnosis and therefore lymph node biopsy should be considered in patients with cervical lymphadenopathy and systemic upset.

\section{REFERENCES:}

1. Harry L. Ioachim, Howard Ratech: Kikuchi-Fujimoto Lymphadenopathy. In: Ioachims Lymph node Pathology. Lippincolt Williams \& Wilkins, third edition, 2001; 219-222.

2. Kikuchi M: Lymphadenitis showing focal reticulum cell hyperplasia with nuclear debris and phagocytosis. Nippon Ketsueki Gakkai Zassi 1972; 35: 378-380.

3. Fujimoto Y. Kozima Y. Yamaguchi K: Cervical subacute necrotizing lymphadentis. A new clinic pathological agent. Naika 1972; 20: 920-927.

4. Louis N, Hanely M, Davidson NM: Kikuchi -Fujimoto disease: a report of two cases and an overview. J Laryngol Otol 1994; 108: 1001-1004.

5. Altuntas F, Sari, Canoz O, Yildiz O, Eser B, Cetin M, Unal A: Kikuchi-Fujimoto disease: a rare but important cause of fever and lymphadenopathy in pregnant women. Am J Haematol 2006; 81 (2): 118-120.

6. Kuo TT: Kikuchi's disease (histiocytic necrotizing lymphadenitis), a clinico-pathologic study of 79 cases with an analysis of histiolytic subtypes, immunohistology and DNA ploidy. Am J Surg Pathol 1995; 798-809.

7. Santana A, Lessa B, Galrao L, Lima I, Santiago M: Kikuchi-Fujimoto's disease associated with systemic lupus erythematous: case report and review of literature. Clin Rheumatal 2005; 24 (1): 60-63.

8. Famularo G. Giustiniani MC, Marasco A, Minisola G, Nicotra GC, De Simone C: Kikuchi-Fujimoto lymphadenitis: case report and literature review. Am J Haemotal 2003; 74 (1): 60-63.

9. Lin HC, Su CY, Huang CC: Kikuchi's disease: a review and analysis of 61 cases. Otolaryngol Head Neck Surg 2003; 128: 650-653.

10. Kucukardali Y, Solmazgul E, Kunter E, Oncul O, Yildirim S, Kaplan M: Kikuchi-Fujimoto Disease : analysis of 244 cases, Clin Rheumatol 2007; 26 (1) : 50-54. Epub 2006 Mar 15. 


\section{CASE REPORT}

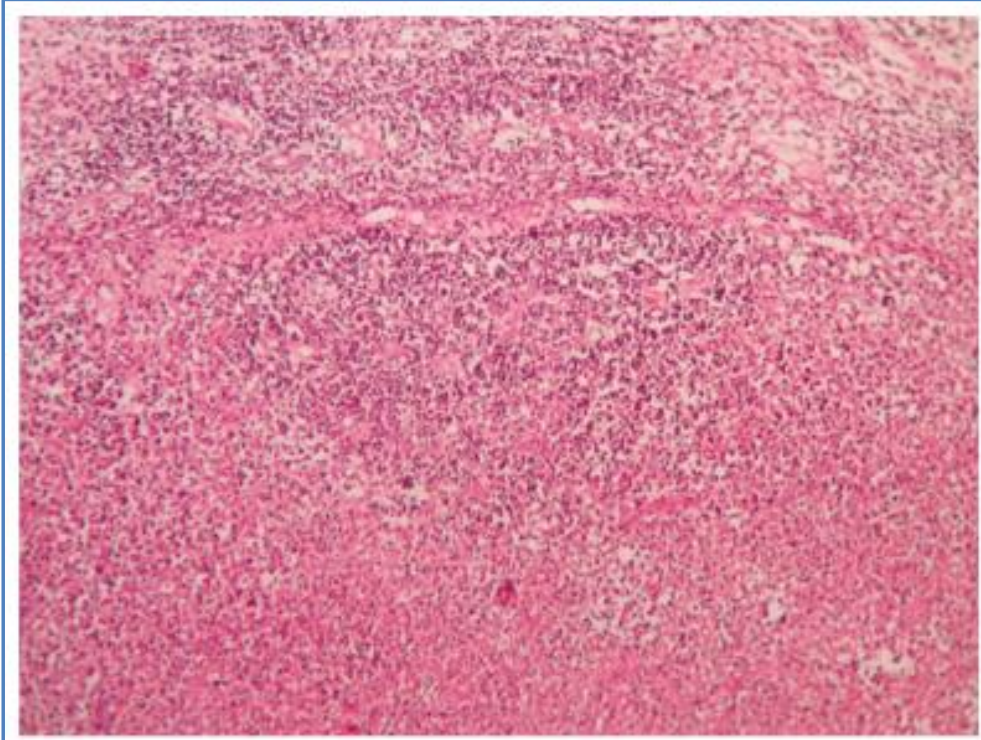

Figure no : 1

Section of lymph node shows paracortical necrosis

(Haematoxylin \& Eosin stained section, X 100 )

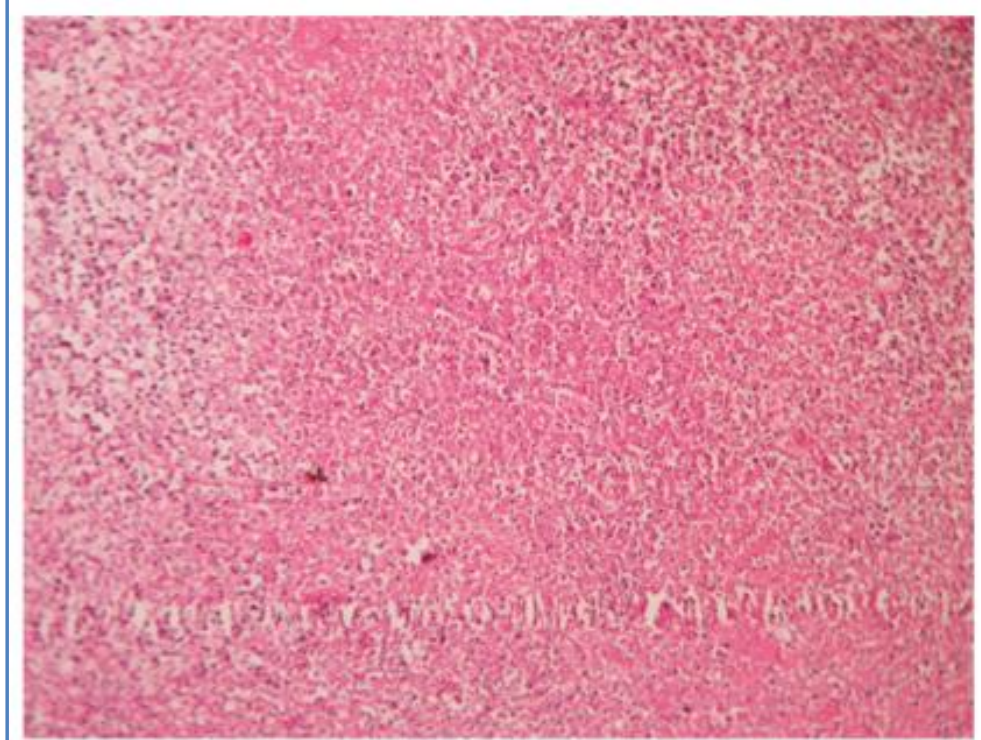

\section{Figure no : 2}

Section of lymph node shows abundant karyorrhectic debris and scattered fibrin deposits.

(Haematoxylin \& Eosin stained section, X 100) 


\section{CASE REPORT}

\section{AUTHORS:}

1. Srinivas Gubbala

2. V. Satyanarayana

\section{PARTICULARS OF CONTRIBUTORS:}

1. Assistant Professor, Department of Pathology, Kamineni Institute of Medical Sciences, Narketpally, Telangana.

2. Professor and HOD, Department of Pathology, Kamineni Institute of Medical Sciences, Narketpally, Telangana.

\section{NAME ADDRESS EMAIL ID OF THE} CORRESPONDING AUTHOR:

Dr. Srinivas Gubbala, Department of Pathology, Kamineni Institute of Medical Sciences, Narketpally, Telangana.

Email: srinivas.path@gmail.com

Date of Submission: 11/09/2014.

Date of Peer Review: 12/09/2014.

Date of Acceptance: 19/09/2014.

Date of Publishing: 29/09/2014. 